\title{
reciturands
}

Revista Cientifica Mundo de la Investigación y el Conocimiento

Alex William Marin Cherrez a ; Marjorie Jacqueline Ramírez Ortiz ${ }^{\text {b; }}$ María Belén Zhindón Astudillo ${ }^{c}$; Javier Fabricio Valdez Zambrano ${ }^{\mathrm{d}}$

Factores de riesgo, Diagnóstico y Tratamiento de la enfermedad de Legg-CalvéPerthes

Risk factors, diagnosis and treatment of Legg-Calvé-Perthes disease

Revista Científica Mundo de la Investigación y el Conocimiento. Vol. 3 núm.3. Esp., noviembre, ISSN: 2588-073X, 2019, pp. 395-420

DOI: $10.26820 /$ recimundo/3.(3.Esp).noviembre.2019.394-420

URL: http://recimundo.com/index.php/es/article/view/604

Código UNESCO: 3205 Medicina Interna

Tipo de Investigación: Artículo de Revisión

(C) RECIMUNDO; Editorial Saberes del Conocimiento, 2019

Recibido: 15/09/2019

Aceptado: 23/10/2019

Publicado: 30/11/2019

Correspondencia: alexmuchinch@gmail.com

\footnotetext{
a. Médico; Investigador Independiente; Guayaquil, Ecuador; alexmuchinch@ gmail.com

b. Magister en Seguridad, Higiene Industrial y Salud Ocupacional; Médico; Investigadora Independiente; Guayaquil, Ecuador; elloreal_22@hotmail.com

c. Médico; Investigadora Independiente; Guayaquil, Ecuador; belenzhindon11@ hotmail.com

d. Médico; Investigador Independiente; Guayaquil, Ecuador; fa_vaza@ @otmail.com
} 


\section{Factores de riesgo, Diagnóstico y Tratamiento de la enfermedad de Legg-}

Calvé-Perthes

Vol. 3, núm. 3 Esp., (2019)

Alex William Marin Cherrez; Marjorie Jacqueline Ramírez Ortiz; María Belén Zhindón Astudillo; Javier Fabricio Valdez Zambrano

\section{RESUMEN}

La enfermedad de Legg-Calvé-Perthes (ELCP/LCPD, por sus siglas en inglés) básicamente se encuentra relacionada a un problema en la cabeza del fémur, debido a la falta de aporte sanguíneo. Históricamente considerada una osteocondrosis, ahora se la conoce como necrosis avascular idiopática de la cabeza femoral en la población pediátrica [NAV]. Etiológicamente, esta complicación aún no ha sido precisada, por lo que es considerada muy extraña. Su incidencia es destacadamente variable entre países, inclusive ha sido así dentro de un mismo país en función del área geográfica. Lo expuesto ha sido el fundamente del interés por desarrollar este tema, y en razón de ello se dispuso el desarrollo de una investigación documental en base a una metodología de revisión enfocada en referir los factores de riesgo, diagnóstico y tratamiento de la ELCP. Los resultados obtenidos tras la consulta en distintas bases de datos arrojaron suficiente información vigente que sirvió no solo para alcanzar el objetivo preestablecido, sino además para definir otros aspectos igualmente relevantes para la comprensión de este tema, tales como sus definiciones, síntomas y etiología relacionada. En definitiva, se logró concluir que, la ELCP aún se concibe como una patología de investigación constante, sobre todo por determinar su etiología definitivamente, entre los factores de riesgo destacan la edad comprendida entre 2 y 8 años, el sexo masculino, la raza blanca, anomalías genéticas, entre otras. El diagnóstico gravita sobre la sospecha sintomatológica, que luego pudiera ser constatado mediante radiografía convencional, en otros casos con gammagrafía y/o ecografía, mientras que en las primeras etapas es mejor la IRM. Entre sus tratamientos ha figurado el consenso generalizado entre los especialistas ortopédicos, quienes defienden la idea de mantener la cabeza femoral dentro del acetábulo como medida estratégica para evitar, entre otras cosas, presiones deformantes sobre la epífisis femoral en la fase de reparación o de plasticidad biológica, la osteoartritis de la cadera con su indeseable cortejo clínico; y promover su esfericidad, asegurar que se mantenga un buen rango de movimiento en la cadera y minimizar los cambios artrósicos secundarios a esta patología.

Palabras Claves: Irrigación de la epífisis capital femoral; Osteocondrosis; Necrosis avascular idiopática; Epífisis femoral; Alteraciones isquémicas. 


\section{Factores de riesgo, Diagnóstico y Tratamiento de la enfermedad de Legg-}

Calvé-Perthes

Vol. 3, núm. 3 Esp., (2019)

Alex William Marin Cherrez; Marjorie Jacqueline Ramírez Ortiz; María Belén Zhindón

Astudillo; Javier Fabricio Valdez Zambrano

\section{ABSTRACT}

Legg-Calvé-Perthes disease (ELCP / LCPD) seems to be a problem in the head of the femur, due to the lack of blood report. Historically it had an osteochondrosis, now it is known as idiopathic avascular necrosis of the femoral head in the pediatric population [NAV]. Etiologically, this complication has not yet been precise, so it is very very strange. Its incidence is remarkably variable between countries, including it has been so within the same country depending on the geographical area. The above has been the basis of interest in developing this issue, and because of that, the development of a documentary investigation based on a review methodology focused on referring the risk factors, diagnosis and treatment of the ELCP. The results obtained after consultation in different databases yielded sufficient current information that served not only to achieve the pre-established objective, but also to define other aspects equally relevant for the understanding of this topic, stories such as limitations, symptoms and related etiology. In conclusion, it is concluded that, the ELCP is still conceived as a constant research pathology, especially for determining its etiology definitively, among the risk factors including the age between 2 and 8 years, the male sex, the white race, genetic abnormalities, among others. The diagnosis gravitates on symptomatic suspicion, which could then be verified by conventional radiography, in other cases with scintigraphy and / or ultrasound, while MRI is better in the early stages. Among its treatments has been the general consensus among orthopedic specialists, who defend the idea of keeping the femoral head inside the acetabulum as a strategic measure to avoid, among other things, deforming pressures on the femoral epiphysis during the repair or biological plasticity phase., osteoarthritis of the hip with its undesirable clinical courtship; and promote its sphericity, ensure that a good range of movement in the hip is maintained and minimize the arthritic changes secondary to this pathology.

Keywords: Femoral capital epiphysis irrigation; Osteochondrosis; Idiopathic avascular necrosis; Femoral epiphysis; Ischemic alterations. 


\section{Factores de riesgo, Diagnóstico y Tratamiento de la enfermedad de Legg-}

\section{Calvé-Perthes}

Vol. 3, núm. 3 Esp., (2019)

Alex William Marin Cherrez; Marjorie Jacqueline Ramírez Ortiz; María Belén Zhindón Astudillo; Javier Fabricio Valdez Zambrano

\section{Introducción.}

Según Padilla, Maya, Bolaños, \& Guerrero (2019) la enfermedad de Legg-Calvé-Perthes (ELCP/LCPD, por sus siglas en inglés) primeramente fue presentada en 1910 por Arthur Legg, en Boston, Estados Unidos de América. De manera indirecta, ese mismo año en Francia, Jacques Calvé proveyó destacados aportes al estudio de Legg y finalmente Georg Perthes hizo lo propio en 1924.

Es un padecimiento ortopédico que afecta el desarrollo de la cadera en los niños, debido a que la irrigación de la epífisis capital femoral presenta alteraciones isquémicas, de origen aún desconocido, que culminan en necrosis. Es una enfermedad rara con una incidencia anual variable (una de cada 250000 personas en Hong Kong; una de cada 18000 en Reino Unido). La ELCP es hasta cinco veces más frecuente en niños que en niñas. (p. 36).

La incidencia de la enfermedad de Perthes varía notablemente entre países e incluso dentro del mismo país en función del área geográfica. Es más frecuente en blancos que en asiáticos o negros. Afecta más a hombres que a mujeres en una proporción de 5/1. En un $15 \%$ de los casos la afectación es bilateral y asimétrica. (Mínguez \& Salom, 2015).

Particularmente, Rampal, Clément, \& Solla (2017) dicen que esta patología constituye la necrosis de la cabeza femoral avascular idiopática en la población pediátrica [NAV] y afligiendo a niños de entre 2 a 10 años de edad. De la misma manera alegan que no es bien conocida la etiología precisa de esta complicación.

La epífisis femoral afectada sufre diversos grados de necrosis auto limitada, lo que 


\section{Factores de riesgo, Diagnóstico y Tratamiento de la enfermedad de Legg-}

Calvé-Perthes

Vol. 3, núm. 3 Esp., (2019)

Alex William Marin Cherrez; Marjorie Jacqueline Ramírez Ortiz; María Belén Zhindón Astudillo; Javier Fabricio Valdez Zambrano

conduce a un amplio espectro de patología y evolución, desde formas leves sin secuelas hasta deformidades graves con un rango de movimiento limitado y osteoartritis temprana de la articulación de la cadera. Clínicamente, el LCPD generalmente se presenta con cojera y dolor localizado en el muslo y / o la rodilla, que ocurre después de una actividad física sostenida en un niño sano y / o déficits en la abducción y rotación interna, y marcha de Trendelenburg en etapas avanzadas. La radiografía simple es el procedimiento de diagnóstico adicional más actual para evaluar la etapa de la enfermedad, la contención de la cabeza femoral en el acetábulo y el grado de afectación del capital. Las indicaciones para el tratamiento dependen principalmente del pronóstico sobre la esfericidad de la cabeza femoral y la congruencia de la cadera al final del crecimiento. (p. 74).

El objeto de la presente revisión estará enfocado en referir cuales son los factores de riesgo, los criterios diagnósticos y de tratamiento asociados a la ELCP, siendo necesario para ello, proceder previamente a definir algunas de sus acepciones aún vigentes, así como también su sintomatología y etiología asociada.

\section{Materiales y Métodos.}

Las herramientas y materiales de trabajo que se utilizaron en el desarrollo de este trabajo investigativo fueron: computadores personales con conexión a internet y un cúmulo de contenidos cientificoacadémicos variado, con los que se persiguió el objetivo aportar información de precisa y vigente respecto a los Factores de riesgo, Diagnóstico y Tratamiento de la enfermedad de Legg-Calvé-Perthes. Para ello se determinó efectuar un trabajo investigativo de diseño documental, de tipo no experimental, a nivel descriptivo, de corte transeccional y bajo 


\section{Factores de riesgo, Diagnóstico y Tratamiento de la enfermedad de Legg-}

\section{Calvé-Perthes}

Vol. 3, núm. 3 Esp., (2019)

Alex William Marin Cherrez; Marjorie Jacqueline Ramírez Ortiz; María Belén Zhindón Astudillo; Javier Fabricio Valdez Zambrano

una metodología de revisión.

A fines del mencionado objetivo, básicamente se adelantó una búsqueda de literatura científico-académica reciente, usando como expresiones claves "Legg-Calvé-Perthes", "necrosis avascular" y "osteonecrosis de la cabeza femoral en niños", tanto en español como en inglés, en diversas bases de datos, tales como: NCBI, BVS, Intramed, MEDLINEPLUS, SciELO, BASE, entre otras.

El tipo de material que se supuso conseguir fue el relativo a: artículos científicos, guías clínicas, e-books, consensos, protocolos, tesis de posgrado y doctorado, ensayos clínicos, noticias científicas, boletines y/o folletos de organismos nacionales e internacionales, públicos o privadas con reconocimiento en el área de la salud, medicina, científica y académica; así como también otros tipos de documentos e informaciones considerados (a criterio del equipo) como de utilidad científico-académica por el valor de la evidencia contenida en los mismo, que, primordialmente, estuvieran accesibles de manera completa y gratuita, y además, que hubiesen sido publicados en los últimos 10 años (incluyendo el corriente) preferiblemente, lo que significa que, igualmente se consideró hacer algunas excepciones.

En consenso, se desestimó todo aquel material que, en su contenido, se detectó con sesgada evidencia o de un valor de escaso nivel, así como también aquella considerada de poco interés científico-académico, entre esas: cartas al editor, resúmenes, monografías, anotaciones e en algunos casos, inclusive, estudios de cohorte, reportes de casos y controles, series y otros tipos de textos. 


\section{Factores de riesgo, Diagnóstico y Tratamiento de la enfermedad de Legg-}

Calvé-Perthes

Vol. 3, núm. 3 Esp., (2019)

Alex William Marin Cherrez; Marjorie Jacqueline Ramírez Ortiz; María Belén Zhindón Astudillo; Javier Fabricio Valdez Zambrano

Entre las distintas bases de datos utilizadas, la tendencia de los resultados obtenidos estribó entre 16 y 197 fuentes bibliográficas de índole distinta, sin embargo, fue considerablemente mayor en los casos de otras pesquisas complementarias efectuadas sin criterios mayores, que respondían más al interés por hallar toda aquella información consensuadamente entendida como de relevancia para el desarrollo general de la temática planteada.

Estos hallazgos, en conjunto, demostraron que este tema se encuentra lo ampliamente documentado, sin embargo, la necesidad de depurar dichos resultados se hizo evidentemente ineludible, razón por la que, ordenada y progresivamente se fueron aplicando ciertos filtros que, fundamentalmente se encontraban disponibles para su uso en cada base de datos consultada, y que respondían en términos generales a criterios tales como: nivel de acceso al recurso, tipo de literatura, idioma, correlación y/o relevancia temática, fecha (o rango) publicación, especialidad (área) temática, entre otros.

Posterior a los procesos antes descritos, es cuando el equipo procedió con la aplicación de técnicas de estudio, tales como: lectura crítica y análisis de datos, interpretación y discusión de todos los documentos definitivamente escogidos, determinados como evidencia para este trabajo investigativo, sumario que sucesiva y definitivamente resultó en la fundamentación de las ideas referidas, que por cierto en todo momento se hizo bajo completo acuerdo.

Finalmente, se aclara que fue a través del mismo consenso grupal que se incluyó y 
Factores de riesgo, Diagnóstico y Tratamiento de la enfermedad de LeggCalvé-Perthes

Vol. 3, núm. 3 Esp., (2019)

Alex William Marin Cherrez; Marjorie Jacqueline Ramírez Ortiz; María Belén Zhindón Astudillo; Javier Fabricio Valdez Zambrano

excluyó, no solamente cada una de las citas y referencias expuestas, sino también las opiniones divergentes.

\section{Resultados.}

La enfermedad de Legg-Calvé-Perthes [ELCP / LCPD, por sus siglas en inglés] es relacionada a un problema en la cabeza del fémur, que en sí vendría siendo la parte esférica del hueso que encaja en la cavidad de la cadera (Figura 1), debido a la falta de aporte sanguíneo.

Figura 1. Representación de la Cabeza del Fémur normal, con daño y otras partes.
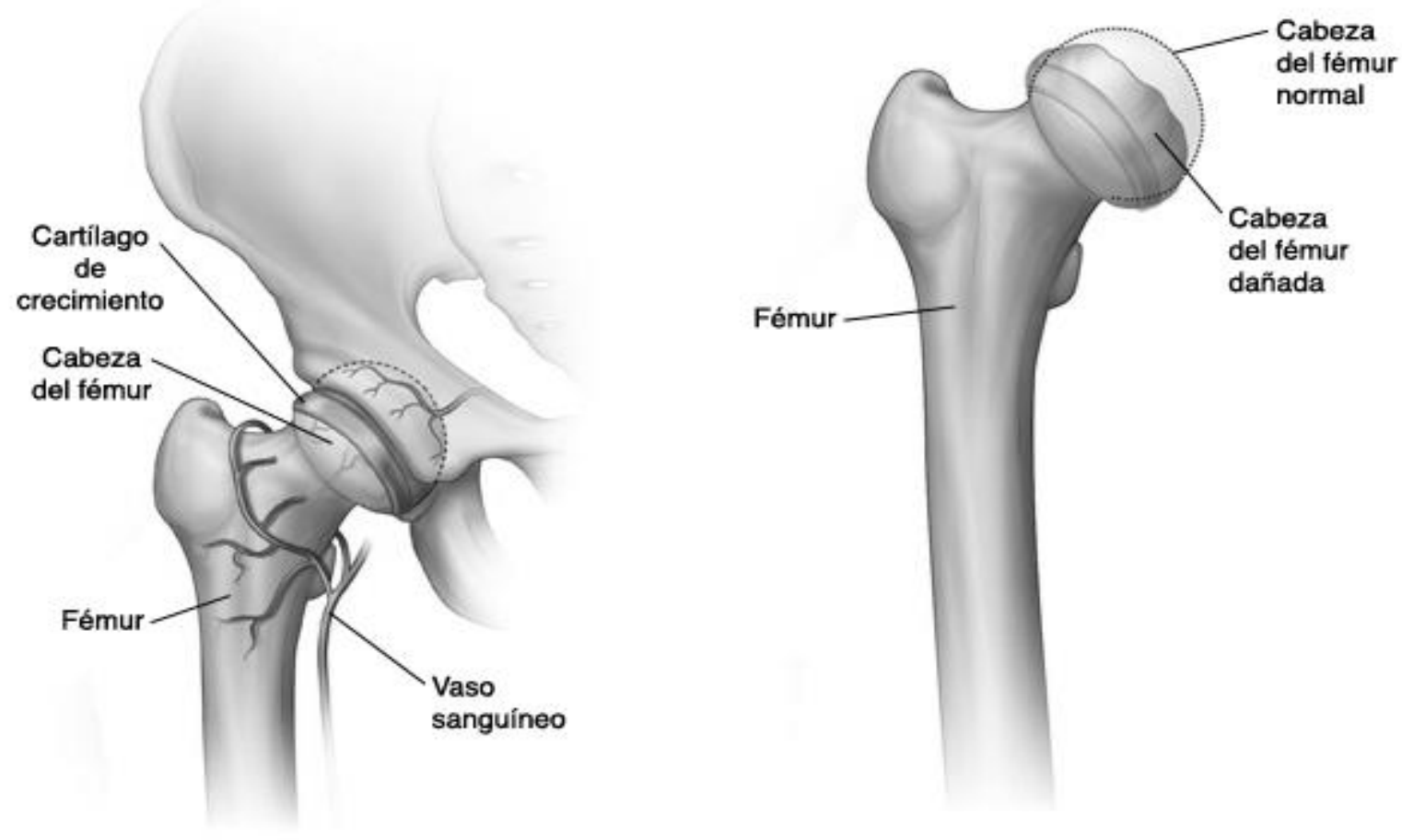

Fuente: (Krames StayWell, 2018) 


\section{Factores de riesgo, Diagnóstico y Tratamiento de la enfermedad de Legg-}

Calvé-Perthes

Vol. 3, núm. 3 Esp., (2019)

Alex William Marin Cherrez; Marjorie Jacqueline Ramírez Ortiz; María Belén Zhindón Astudillo; Javier Fabricio Valdez Zambrano

En la obra de Wiig (2009) se deja claro que la ELCP es un término que se usa para describir la necrosis avascular uni lateral o bilateral (NAV) de la cabeza femoral en los niños.

La incidencia anual varía considerablemente, desde 1/250.000 en Hong Kong y 1/18.000 en Reino Unido, a 1/3.500 por año en las Islas Feroe. La LCPD afecta a niños de edad comprendida entre los 2 y los 12 años, pero es más prevalente en niños de entre 5 y 6 años y más común en varones.

Padilla, Maya, Bolaños, \& Guerrero (2019) en su estudio alegan que esta se define por:

[...] la necrosis en el tejido óseo, cartílago articular con proliferación de condrocitos en capas superficiales y tejido muerto en la capa profunda con posible fractura subcondral del hueso contiguo y rotura de la placa de crecimiento, lo cual progresa a resorción ósea y finalmente a una reosificación. Todo esto se ve reflejado en una radiografía, en la que se presenta la cabeza femoral en forma de hongo con acortamiento y engrosamiento del cuello; remodelación del acetábulo, inclinado con eje acortado; y rarefacción metafisiaria. (p. 36).

La opinión de Pavone (2019) es que esta es una complicada enfermedad de la población pediátrica que afecta la epífisis de la cabeza femoral. Así mismo dejan ver que:

Históricamente considerada una osteocondrosis, ahora se la conoce como necrosis avascular idiopática de la cabeza femoral en la población pediátrica. Entre su prevalencia no hay acuerdo general. Se establece entre 0,4 / 100000 a 29,0 / 100000 niños <15 años de edad con un pico de incidencia en niños de 4 años a 8 años y una relación hombre / mujer de 5:1. Un estudio 


\section{Factores de riesgo, Diagnóstico y Tratamiento de la enfermedad de Legg-}

\section{Calvé-Perthes}

Vol. 3, núm. 3 Esp., (2019)

Alex William Marin Cherrez; Marjorie Jacqueline Ramírez Ortiz; María Belén Zhindón Astudillo; Javier Fabricio Valdez Zambrano

epidemiológico de alto perfil realizado en 2017 con la participación de 2,1 millones de personas intentaron informar una prevalencia más precisa de esta enfermedad. Se encontró una prevalencia general de 9.3 por 100000 sujetos. La relación hombre / mujer fue de 3.1: 1. Aunque el estudio se realizó en Suecia desde 1973 hasta 1993, es una de las fuentes de evidencia más actualizadas de la epidemiología de LCPD.

\section{Síntomas}

Los síntomas iniciales son generalmente: cojera, dolor en la cadera, muslo o rodilla y movilidad de cadera reducida. Más tarde, durante el curso de la enfermedad, puede observarse una discrepancia de la longitud de la pierna, así como una atrofia muscular alrededor de la cadera. La fase activa de la enfermedad puede durar varios años y se caracteriza por la necrotización parcial o total de la cabeza femoral y su progresiva deformación. Esta fase está seguida por la formación de hueso nuevo (re-osificación) en la epífisis y la eventual curación. La deformación final puede variar de una configuración casi normal de la articulación, a una deformación extensa con aplanamiento y subluxación graves de la cabeza femoral, alargamiento del cuello femoral y deformación y displasia del acetábulo, que a su vez puede conducir a una osteoartritis de aparición temprana. (Wiig, 2009).

De acuerdo con Hernández, Romero, Osorto, \& Madrid (2014), la sintomatología y lo que se detecta tras la exploración física será muy variada en relación a la etapa en que se ubique esta complicación, que de conformidad a su avance en el tiempo, se divide en cuatro fases que son: 


\section{Factores de riesgo, Diagnóstico y Tratamiento de la enfermedad de Legg-}

Calvé-Perthes

Vol. 3, núm. 3 Esp., (2019)

Alex William Marin Cherrez; Marjorie Jacqueline Ramírez Ortiz; María Belén Zhindón Astudillo; Javier Fabricio Valdez Zambrano

\section{Inicial duración promedio de 6 meses (1-14 meses)}

II. Fragmentación de 8 meses (2-35 meses)

\section{Reosificación o cicatrización de 51 meses (2-12 meses)}

IV. Residual o remodelación continua hasta la madurez esquelética.

En las primeras fases de la enfermedad la presentación más frecuente suele ser la de un niño con cojera o con dolor progresivo en la ingle, el muslo o la rodilla. En la exploración física encontraremos una hipotrofia o atrofia del muslo, los gemelos y la nalga del miembro inferior. (p. 18-19).

En el mismo orden de ideas, el personal del Hospital Infantil de Stanford de Estados Unidos de América describe que en la fase I (inicial). se manifiesta la inflamación, rigidez y dolor en las articulaciones de la cadera. En algunas partes del hueso se genera la necrosis del tejido óseo. Radiológicamente, la cabeza del fémur se ve menos redondeada. Toda esta esta fase puede durar desde unos meses hasta un año. En la fase II (fragmentación), que en tiempo puede ocurrir desde uno hasta tres años, las células óseas muertas serán eliminadas por el cuerpo y las reemplazará por células óseas nuevas y más sanas. La forma redondeada de cabeza femoral comenzará nuevamente a generarse mientras que se mantiene la irritación al igual que dolor en las articulaciones. La fase III (osificación) que igualmente podría durar de uno a tres años, se caracterizará por continuación de la confección redondeada la cabeza del fémur, así como de nuevo tejido óseo. Para fase IV (curación), las nuevas células óseas serán reemplazadas por las células normales, pudiendo este proceso manifestarse durante varios años hasta completar el 


\section{Factores de riesgo, Diagnóstico y Tratamiento de la enfermedad de Legg-}

\section{Calvé-Perthes}

Vol. 3, núm. 3 Esp., (2019)

Alex William Marin Cherrez; Marjorie Jacqueline Ramírez Ortiz; María Belén Zhindón Astudillo; Javier Fabricio Valdez Zambrano

proceso de curación. (Lucile Packard Children’s Hospital Stanford, 2019).

En este nivel se vuelve importante mencionar el aporte de Vargas \& Martínez (2012) debido a que refiere que fue (Herring, 1992) quien creó "una clasificación basada en los cambios radiológicos del segmento lateral de la cabeza femoral en fase de fragmentación" (Figura 2.) determinando entonces:

[...] 3 grupos $[A, B$ y $C$ ] según la altura de dicho segmento, encontrando una correlación clara con el pronóstico de la enfermedad ya que, a mayor altura, mayor protección contra el derrumbamiento, y cuando correlacionamos los hallazgos radiológicos con la edad de aparición de la enfermedad el nivel de predicción es mejor. (p. 169).

Los mismos autores parten del hecho de que, según la mencionada clasificación, lo que se considera «Normal» es la "división funcional de la cabeza del fémur en pilares lateral, central y medial". Por lo tanto, describen que:

Grupo A: La altura del pilar lateral es la normal. Puede haber radiolucidez en pilares central y medial, pero sin pérdida de altura del pilar lateral. Buen pronóstico.

Grupo B: Pérdida de altura del pilar lateral pero menor del $50 \%$ de su altura normal. El segmento central puede estar más hundido.

Grupo C: El colapso del pilar lateral es superior al 50\% de su altura normal. Los pilares central y medial pueden haber perdido su altura normal, pero en menor grado que 
Factores de riesgo, Diagnóstico y Tratamiento de la enfermedad de Legg-

Calvé-Perthes

Vol. 3, núm. 3 Esp., (2019)

Alex William Marin Cherrez; Marjorie Jacqueline Ramírez Ortiz; María Belén Zhindón Astudillo; Javier Fabricio Valdez Zambrano

el pilar lateral. Mal pronóstico. [...] Sobre esta clasificación algunos autores han creído conveniente agregar un cuarto grupo denominado «grupo B/C» para casos limítrofes en los que el pilar lateral conserva la altura del 50\%, pero el segmento remanente tiene muchas irregularidades radiográficas1,6. La clasificación del pilar lateral de Herring tiene un mayor valor pronóstico y menor variabilidad interobservador que la clasificación de Catterall2,8, por lo que hoy día es la clasificación más utilizada. (Vargas \& Martínez, 2012, p. 169 - 170).

Figura 2. Clasificación de Herring (Área necrótica en la sombra más oscura)
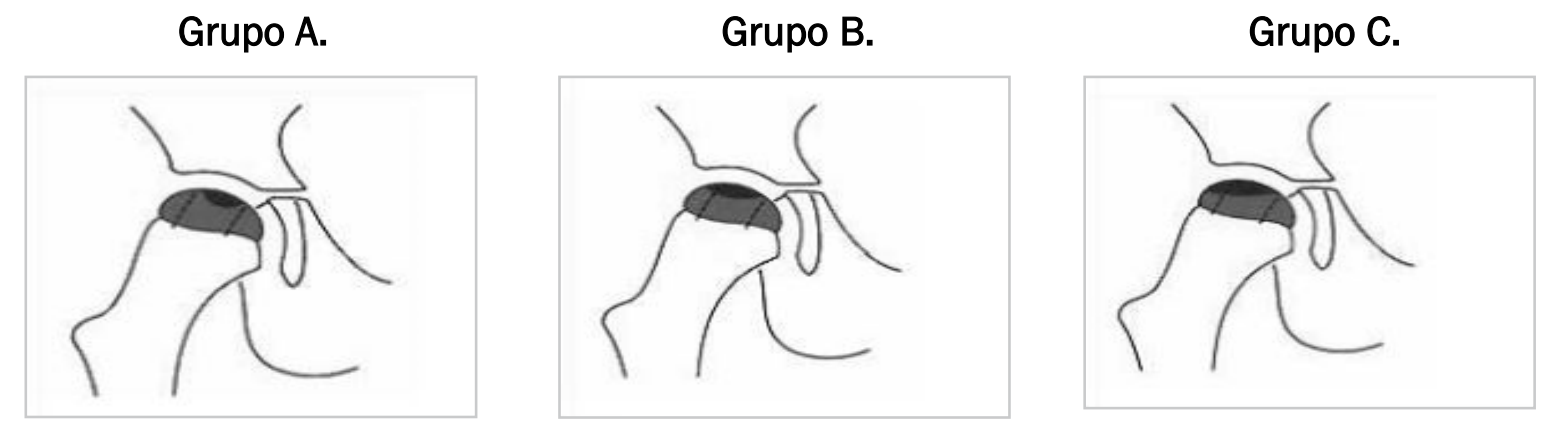

\section{Etiología}

La etiología de la LCPD permanece desconocida. En general, se cree que la deformación puede estar causada por uno o más infartos de la cabeza femoral, secundarios a la interrupción del aporte vascular. Sin embargo, también existen otras teorías sobre la causa de la interrupción. (Wiig, 2009). 


\section{Factores de riesgo, Diagnóstico y Tratamiento de la enfermedad de Legg-}

\section{Calvé-Perthes}

Vol. 3, núm. 3 Esp., (2019)

Alex William Marin Cherrez; Marjorie Jacqueline Ramírez Ortiz; María Belén Zhindón Astudillo; Javier Fabricio Valdez Zambrano

Este aspecto ha sido destacadamente investigado por Pavone et al. (2019) ya que, respecto a la etiología de la LCPD ha indicado que aún se desconoce fundamentalmente.

Los tratadistas explican que ello es debido a la variada prevalencia indicada en importantes estudios, lo que entonces ha abierto el debate en cuanto a la evaluación de los "posibles factores ambientales y sociales involucrados en la etiología de la enfermedad". En base a ello, refieren, tres estudios evidenciaron (aún no definitivamente) una posible relación entre la reducción de la ocurrencia y los cambios en el estilo de vida en los últimos años, la exposición a factores de riesgo ambientales como el tabaquismo, la osificación hipofisaria tardía, el bajo peso al nacer, la privación infantil y la obesidad.

Continúan refiriendo que, otros estudios demostraron más fehacientemente la relación entre la incidencia de LCPD en niños y el papel de la privación social y económica.

Esto se propuso como una posible consecuencia de dos factores: bajo peso al nacer y hábitos de fumar. Además, el tabaquismo materno se asocia con un bajo peso al nacer. Por lo tanto, el bajo peso al nacer a menudo podría asociarse falsamente con LCPD debido al hábito de fumar típicamente presente en estas familias, que es la causa más probable de la mayor incidencia de LCPD. Por estas razones, el papel del bajo peso al nacer carece de evidencia sólida, y el hábito de fumar, que es más común en las familias desfavorecidas socioeconómicamente, puede desempeñar un papel más importante en la etiopatogenia de la LCPD. Esto fue confirmado por estudios que investigaron la asociación entre fumar y LCPD, que informaron un aumento significativo del riesgo en fumadores y familiares expuestos al humo. El mecanismo 


\section{Factores de riesgo, Diagnóstico y Tratamiento de la enfermedad de Legg-}

Calvé-Perthes

Vol. 3, núm. 3 Esp., (2019)

Alex William Marin Cherrez; Marjorie Jacqueline Ramírez Ortiz; María Belén Zhindón Astudillo; Javier Fabricio Valdez Zambrano

propuesto es el daño dependiente del humo del endotelio de los vasos, que en última instancia podría conducir a un infarto hipofisario. (p.159).

Así mismo, los autores han indicado que reciente evidencia señala a la obesidad como un factor de riesgo importante.

La leptina es una hormona que se expresa en el tejido adiposo y, en niveles más bajos, en el epitelio gástrico y la placenta asociados con la obesidad y el metabolismo óseo. Se ha informado que tanto la leptina como la obesidad se asocian positivamente con la gravedad de la LCPD. La mayoría de los humanos obesos tienen concentraciones de leptina en plasma muy altas, lo que sugiere que son resistentes a sus efectos anoréxicos y metabólicos. En base a estos hallazgos, Bartell et al. 2011 realizó un estudio que incluyó la administración intracerebroventricular y subcutánea de leptina en ratones ob / ob con deficiencia de leptina, investigando su efecto sobre los tejidos óseos y musculares. En ambos experimentos, la leptina tuvo un papel clave en la disminución del peso corporal, la ingesta de alimentos y la grasa corporal y en el aumento de la masa muscular, la densidad mineral ósea, el contenido mineral óseo, el área ósea, el número de adipocitos de médula y la tasa de aposición mineral. Por lo tanto, la administración de leptina parecía ser realmente efectiva en el metabolismo óseo de pacientes obesos. Siguiendo esta justificación, Zhou et al. 2015 probó estos efectos en un modelo de rata obesa LCPD. Seis semanas después de la cirugía indujo necrosis avascular de la cabeza femoral, se realizaron evaluaciones radiológicas e histomorfométricas. Las radiografías mostraron una mejor preservación de la arquitectura de la cabeza femoral en el grupo 


\section{Factores de riesgo, Diagnóstico y Tratamiento de la enfermedad de Legg-}

\section{Calvé-Perthes}

Vol. 3, núm. 3 Esp., (2019)

Alex William Marin Cherrez; Marjorie Jacqueline Ramírez Ortiz; María Belén Zhindón Astudillo; Javier Fabricio Valdez Zambrano

tratado con leptina. La histología y la inmunohistoquímica revelaron que el grupo de leptina había aumentado significativamente la proliferación osteoblástica y la vascularización en las cabezas femorales infartadas en comparación con los grupos de control. El mecanismo propuesto está relacionado tanto con una acción directa de la leptina sobre el metabolismo óseo como con una acción indirecta a través de la regulación positiva de VEGF. En particular, la leptina actúa fisiológicamente a través de las vías MAPK / ERK 1/2 y PI-3K / AKT1 y una serie de factores de transcripción como HIF-1 $\alpha$. La evidencia emergente respalda cómo la leptina y la obesidad pueden desempeñar un papel en la etiopatogenia de LCPD. Recomendamos encarecidamente realizar más estudios sobre la leptina y la obesidad, asociados con sus efectos del metabolismo óseo, en pacientes con LCPD. Estos estudios deben realizarse como una opción terapéutica y como un posible actor en la etiología de la enfermedad. La diferencia en la incidencia entre sujetos masculinos y femeninos inicialmente se pensó que dependía de la etiopatogenia diferente de la enfermedad. En particular, los estudios pioneros sobre estas diferencias informaron una presentación y un pronóstico más severos en pacientes femeninos que en sujetos masculinos. Sin embargo, estudios epidemiológicos recientes de alto perfil no informaron diferencias significativas en la presentación clínica, el resultado y el pronóstico entre niños y niñas. Por lo tanto, la relación LCPD hombre / mujer parece no estar asociada con diferentes presentaciones clínicas y no debe ser parte del algoritmo de tratamiento clínico. (p. 159).

En definitiva, estos expertos refieren que entre otras fortalezas de su estudio destacan el 


\section{Factores de riesgo, Diagnóstico y Tratamiento de la enfermedad de Legg-}

Calvé-Perthes

Vol. 3, núm. 3 Esp., (2019)

Alex William Marin Cherrez; Marjorie Jacqueline Ramírez Ortiz; María Belén Zhindón Astudillo; Javier Fabricio Valdez Zambrano

hecho de: haber efectuado una exhaustiva búsqueda por medio de la cual pudieron identificar todos los estudios importantes sobre asociación genética, la comorbilidad más frecuente y la posible hipótesis etiológica. "Por lo tanto, la evaluación del riesgo de sesgo mostró un riesgo general moderado que podría influir en nuestro análisis."

De la misma manera reconocen que:

La literatura disponible sobre la etiología de LCPD presenta limitaciones importantes en términos de gran heterogeneidad y falta de estudios de alto perfil. Aunque muchos estudios se centraron en los antecedentes genéticos, biomecánicos y radiológicos de la enfermedad, no hay consenso sobre uno o múltiples actores principales de la etiopatogenia. Si bien la obesidad, la exposición al tabaquismo y la privación infantil parecen estar asociadas con la etiología del LCPD, se necesitan más estudios para comprender la génesis compleja y multifactorial de la necrosis avascular que caracteriza la enfermedad. (p. 160).

Factores de Riesgo

- La edad. Aunque la enfermedad de Legg-Calvé-Perthes puede afectar a los niños de casi cualquier edad, es más frecuente que comience entre las edades de 4 y 8 años.

- El sexo del niño. Legg-Calvé-Perthes es cinco veces más frecuente entre los niños que entre las niñas.

- Raza. Los niños de raza blanca son más propensos a manifestar esta alteración que 


\section{Factores de riesgo, Diagnóstico y Tratamiento de la enfermedad de Legg-}

\section{Calvé-Perthes}

Vol. 3, núm. 3 Esp., (2019)

Alex William Marin Cherrez; Marjorie Jacqueline Ramírez Ortiz; María Belén Zhindón Astudillo; Javier Fabricio Valdez Zambrano

aquellos de raza negra o afroamericana.

- Mutaciones genéticas. En una pequeña cantidad de casos, la enfermedad de Legg-

Calvé-Perthes parece estar relacionada con mutaciones en ciertos genes. (Mayo Clinic, 2018)

Se han sugerido otros factores implicados: retraso en la maduración esquelética, déficit del crecimiento y crecimiento desproporcionado, estatura baja, bajo peso al nacer, privación social y económica y traumatismo, así como una asociación con anomalías congénitas.

También se ha sugerido que los trastornos del sistema de coagulación pueden causar trombofilia y/o hipofibrinolisis y conducir a la oclusión trombótica venosa, con posterior NAV (necrosis avascular) de la cabeza femoral en los niños. (Wiig, 2009)

\section{Diagnóstico}

El diagnóstico se realiza por radiografía convencional con proyecciones frontal y lateral. La gammagrafía y la ecografía pueden ser útiles en algunos casos y la IRM [imagen por resonancia magnética] puede ser útil en las primeras etapas de la enfermedad para distinguir la LCPD de otros trastornos de la cadera. El diagnóstico diferencial incluye la displasia de Meyers, la displasia epifisiaria múltiple y la displasia espondiloepifisiaria. (Wiig, 2009)

Pessler (2017) particularmente afirma que:

El diagnóstico de la enfermedad de Legg-Calvé-Perthes se sospecha por los síntomas. Por lo general, se obtienen rayos X y, si se necesita, se realiza una RM para 


\section{Factores de riesgo, Diagnóstico y Tratamiento de la enfermedad de Legg-}

Calvé-Perthes

Vol. 3, núm. 3 Esp., (2019)

Alex William Marin Cherrez; Marjorie Jacqueline Ramírez Ortiz; María Belén Zhindón Astudillo; Javier Fabricio Valdez Zambrano

confirmar el diagnóstico y el alcance de la lesión. Al principio, las radiografías pueden no ser diagnósticas, porque son normales o muestran mínimo aplanamiento. Las radiografías posteriores pueden mostrar fragmentación de la cabeza femoral, que contiene zonas radiolúcidas y de esclerosis. En los casos bilaterales o familiares, se impone un estudio radiográfico del esqueleto para descartar trastornos esqueléticos hereditarios, en particular la displasia epifisaria múltiple, porque el pronóstico y el tratamiento óptimo son diferentes. También deben descartarse el hipotiroidismo, la anemia drepanocítica y los traumatismos.

\section{Tratamiento}

Para Wiig (2009) el objetivo principal es el de contener la cabeza femoral en el acetábulo, usando un aparato ortopédico o por medio de intervenciones quirúrgicas (osteotomía femoral o pélvica).

Un estudio reciente sugiere que la osteotomía femoral da resultados significativamente mejores que el tratamiento con aparatos ortopédicos (concretamente, la órtesis de abducción de tipo «Scottish Rite » (Wiig, 2009).

Por su parte, Mínguez \& Salom (2015) aseguran que aún es debatido el tratamiento idóneo para la enfermedad de Legg-Calvé-Perthes, sin embargo, en la comunidad ortopédica se ha dado un consenso generalizado que respalda la idea de "la contención de la cabeza femoral como medida para promover su esfericidad y minimizar los cambios artrósicos secundarios a esta patología. Esta contención puede obtenerse por métodos conservadores o quirúrgicos". 


\section{Factores de riesgo, Diagnóstico y Tratamiento de la enfermedad de Legg-}

\section{Calvé-Perthes}

Vol. 3, núm. 3 Esp., (2019)

Alex William Marin Cherrez; Marjorie Jacqueline Ramírez Ortiz; María Belén Zhindón Astudillo; Javier Fabricio Valdez Zambrano

(Mínguez \& Salom, 2015).

En esto concuerdan Hernández, Romero, Osorto, \& Madrid (2014), ya que respecto a la contención de la epífisis femoral dentro del acetábulo explican que: "[...] tiene como finalidad evitar presiones deformantes sobre la epífisis femoral en la fase de reparación o de plasticidad biológica, para prevenir la osteoartritis de la cadera con su indeseable cortejo clínico." (p. 19).

Es necesario hacer una distinción referente a lo antes dicho respecto a la contención de la cabeza femoral en el acetábulo, por ello es oportuno referir la obra de Hunt et al. (2012) quienes describen que dichos métodos (invasivos o no) están referidos a aquellos:

I. No quirúrgicos: por tratarse de diversos aparatos entre los que destacan "el de Toronto, el de Birmingham, el Newington, el de Tachdjian y el de Atlanta" que tras su uso han resultado en demostrar distintos grados de éxito. No obstante, aclaran que en la actualidad "estos métodos de tratamiento están en desuso, ya que varios estudios han demostrado que no alteran favorablemente la evolución natural de la enfermedad" aunado a esto se manifiesta la incomodidad de su utilización, su necesario uso prolongado, la dificultad para determinar el inicio y fin de su implementación, y el marcado rechazo manifestado tanto por el paciente como por sus familiares en cuanto al uso de este tipo de aparatos.

II. Quirúrgicos: Mayormente indicados para aquellos casos de pacientes con mayor grado de afección en las caderas y pacientes con edad superior a los 6 años, siendo necesario en ambos efectuar un manejo adecuado de los efectos asociados. La Osteotomía femoral 


\section{Factores de riesgo, Diagnóstico y Tratamiento de la enfermedad de Legg-}

Calvé-Perthes

Vol. 3, núm. 3 Esp., (2019)

Alex William Marin Cherrez; Marjorie Jacqueline Ramírez Ortiz; María Belén Zhindón Astudillo; Javier Fabricio Valdez Zambrano

proximal y la Osteotomía de Salter vendrían siendo los dos procedimientos más frecuentemente ejecutados. El primero de ellos definido como la osteotomía varizante de la porción proximal del fémur, se refiere a centrar la cabeza femoral en la profundidad del acetábulo mientras mantiene el miembro alineado en posición de carga; mientras que el segundo:

[...] consiste en efectuar un corte horizontal del iliaco, para reorientar el fragmento distal de la hemipelvis, trasladando el cótilo hacia abajo (aducción) y delante (extensión), usando como pivote de rotación a la sínfisis púbica. El efecto que se obtiene es cubrir la cabeza femoral por arriba, por lateral (aproximadamente $15^{\circ}$ ) y por anterior (aproximadamente $25^{\circ}$ ). (p. 11).

Estos igualmente aclaran que se dan casos en los que la combinación de las referidas osteotomías se vuelve necesaria para obtener "una mayor cobertura de la cabeza femoral", y ello comúnmente ocurre en pacientes de más de 9 años de edad, con subluxación lateral de la cabeza femoral. A la par refieren que existen otros procedimientos que se pudieran llevar a cabo, tales como: Artroplastía tipo Shelf: formalmente definida como método de tratamiento de "salvamento" en los casos impropios, facilitando el recubrimiento de la porción más anterolateral de la cabeza femoral y previniendo la subluxación o el crecimiento lateral de la epífisis, también, en la subluxación lateral de la cabeza, la inadecuada cobertura o la cadera en "bisagra" asociada a las formas severas; la Osteotomía de Chiari, que favorece la cobertura lateral de la cabeza deformada y/o aplastada, más no subyuga el rozamiento lateral a la abducción y puede incrementar la insuficiencia glútea; la Queilectomía, intervención anteriormente utilizada que 


\section{Factores de riesgo, Diagnóstico y Tratamiento de la enfermedad de Legg-}

\section{Calvé-Perthes}

Vol. 3, núm. 3 Esp., (2019)

Alex William Marin Cherrez; Marjorie Jacqueline Ramírez Ortiz; María Belén Zhindón Astudillo; Javier Fabricio Valdez Zambrano

significa la sustracción de fragmentos de la cabeza femoral que protruyen en la porción anterolateral, con la idea de mejorar la movilidad y evitar el fenómeno de abducción en bisagra. (p. 12)

Kaneshiro (2017) coincide en el hecho de que, tratar de contener la cabeza femoral dentro del acetábulo, es el objetivo del tratamiento de esta condición, con la finalidad de asegurar que se mantenga un buen rango de movimiento en la cadera. La estrategia para tratar esta patología incluye:

- Un corto período de reposo en cama para aliviar el dolor intenso.

- Limitar la cantidad de peso que se coloca en la pierna mediante la restricción de actividades como correr.

- Fisioterapia para ayudar a mantener los músculos de la pierna y la cadera fuertes.

- Tomar medicamento antiinflamatorio, como ibuprofeno, para aliviar la rigidez en la articulación de la cadera.

- Usar una férula o un dispositivo ortopédico para ayudar con la contención.

- Usar muletas o un caminador.

Cuando otros tratamientos no funcionen, es posible que se necesite una intervención quirúrgica que puede tratarse de desde un alargamiento de un músculo de la ingle hasta una osteotomía. Dependerá de la gravedad de la complicación y de la forma de la cabeza de la articulación de la cadera la elección precisa del tipo de operación. 


\section{Factores de riesgo, Diagnóstico y Tratamiento de la enfermedad de Legg-}

Calvé-Perthes

Vol. 3, núm. 3 Esp., (2019)

Alex William Marin Cherrez; Marjorie Jacqueline Ramírez Ortiz; María Belén Zhindón Astudillo; Javier Fabricio Valdez Zambrano

Para Pessler (2017) el tratamiento se resume a: reposo e inmovilización, por una parte, y ocasionalmente, cirugía. Expone que el tratamiento ortopédico se trata de "reposo en cama prolongado, tracción móvil, cabestrillos y escayolas y férulas en abducción para contener la cabeza femoral." Refiere que otros expertos proponen la osteotomía subtroncantérea con fijación interna y deambulación precoz. Por otra parte, indica que a pesar de lo efectivo que ha evidenciado ser en pruebas iniciales el uso de los bifosfonatos, estos estudios aún no son concluyentes.

Sin tratamiento, la evolución suele ser prolongada, pero autolimitada (en general, 2-3 años). Cuando la enfermedad por fin se vuelve quiescente, la distorsión residual de la cabeza femoral y el acetábulo predispone a artrosis degenerativa secundaria. Con tratamiento, las secuelas son menos graves. Los niños pequeños y los niños con menos destrucción de la cabeza femoral cuando se diagnostica tienen los mejores resultados.

\section{Conclusiones.}

Es evidente que la ELCP sigue concibiéndose como una patología en constante investigación, sobre todo en el hecho de tratar de determinar definitivamente su etiología, para así favorecer un mejor manejo de ella, en general.

Entre los factores de riesgo asociados destacaron el de la edad (de 2 a 8 años), sexo masculino, raza blanca, anomalías genéticas (mutaciones genéticas), retraso en la maduración esquelética, déficit del crecimiento y crecimiento desproporcionado, estatura baja, bajo peso al nacer (BPN), privación social y económica y traumatismo y trastornos del sistema de 


\section{Factores de riesgo, Diagnóstico y Tratamiento de la enfermedad de Legg-}

\section{Calvé-Perthes}

Vol. 3, núm. 3 Esp., (2019)

Alex William Marin Cherrez; Marjorie Jacqueline Ramírez Ortiz; María Belén Zhindón Astudillo; Javier Fabricio Valdez Zambrano

coagulación.

El diagnóstico se basa en una sospecha sintomatológica, que luego pudiera constatarse mediante una secuencia de radiografías convencionales, aunque en algunos casos se procede con la gammagrafía y/o ecografía. Ahora bien, en las primeras etapas es mejor la IRM puesto que es posible que los cambios en las primeras radiografías sean poco detectables, casos estos en los que se retarda el diagnóstico por algunos meses, inclusive existiendo manifestaciones sintomatológicas típica de la patología.

Finalmente, basado en el referido consenso generalizado de la especialidad ortopédica relativo a la idea de mantener la cabeza femoral dentro del acetábulo como medida estratégica para evitar presiones deformantes sobre la epífisis femoral en la fase de reparación o de plasticidad biológica, prevenir la osteoartritis de la cadera con su indeseable cortejo clínico, promover su esfericidad, asegurar que se mantenga un buen rango de movimiento en la cadera y minimizar los cambios artrósicos secundarios a esta patología.

\section{Bibliografía.}

Hernández, S., Romero, B., Osorto, R., \& Madrid, A. (2014). Enfermedad de Legg-CalvéPerthes. Revista Médica Hondureña, 82(2), 18-20.

Hunt, N., García, C., Abiuso, V., Hernández, J., Gomez, R., \& Rostion, C. (2012). Enfermedad de “Legg- Calvé-PERTHES” en la infancia. Revista Pediatría Electrónica, 9(2), 1-18.

Kaneshiro, N. (5 de Septiembre de 2017). Enfermedad de Legg-Calve-Perthes. Obtenido de Medline Plus: https://medlineplus.gov/spanish/ency/article/001264.htm

Krames StayWell. (2018). Cuando su hijo tiene la enfermedad de Legge Calvé Perthes. Recuperado el 23 de 10 de 2019, de M health: https://www.mhealth.org/patienteducation/89364 


\section{Factores de riesgo, Diagnóstico y Tratamiento de la enfermedad de Legg-}

Calvé-Perthes

Vol. 3, núm. 3 Esp., (2019)

Alex William Marin Cherrez; Marjorie Jacqueline Ramírez Ortiz; María Belén Zhindón

Astudillo; Javier Fabricio Valdez Zambrano

Lucile Packard Children's Hospital Stanford. (2019). Enfermedad de Legg-Calvé-Perthes. Obtenido de Stanford Children's Health : https://www.stanfordchildrens.org/es/topic/default?id=legg-calv-perthesdisease-90P05879

Mayo Clinic. (20 de Noviembre de 2018). Enfermedad de Legg-Calvé-Perthes. Obtenido de Mayo Clinic: https://www.mayoclinic.org/es-es/diseases-conditions/legg-calve-perthesdisease/symptoms-causes/syc-20374343

Mínguez, M., \& Salom, M. (2015). Enfermedad de Legg-Calvé-Perthes. Conceptos actuales. Revista Española de Cirugía Osteoarticular, 50(261), 79-82.

Padilla, F., Maya, L., Bolaños, G., \& Guerrero, D. (2019). El posible origen de la enfermedad de Legg-Calvé-Perthes. Revista Médica del Instituto Mexicano del Seguro Social, 57(1), 3641.

Pavone, V., Chisari, E., Vescio, A., Lizzio, C., Sessa, G., \& Testa, G. (18 de Marzo de 2019). Etiología de la enfermedad de Legg-Calvé-Perthes: una revisión sistemática. World Journal of Orthopedics, 10(3), 145-165.

Pessler, F. (Febrero de 2017). Enfermedad de Legg-Calvé-Perthes. Obtenido de Manual MSD: https://www.msdmanuals.com/es/professional/pediatr\%C $3 \% \mathrm{ADa} /$ trastornos$\% \mathrm{C} 3 \% \mathrm{~B} 3$ seos-en-ni\%C $3 \% \mathrm{~B} 1$ os/enfermedad-de-legg-calv\%C3\%A9-perthes

Rampal, V., Clément, J.-L., \& Solla, F. (Abril de 2017). Enfermedad de Legg-Calvé-Perthes: clasificaciones y factores pronósticos. Clinical Cases in Mineral Bone Metabolism, 14(1), 74-82.

Vargas, I., \& Martínez, Ó. (Abril de 2012). Enfermedad de Legg-Calvé-Perthes. Revisión actualizada. Semergen, 38(3), 167-174.

Wiig, O. (Marzo de 2009). Enfermedad de Legg-Calvé-Perthes. Obtenido de Orphanet: https://www.orpha.net/consor/cgi-bin/OC_Exp.php?Lng=ES\&Expert=2380 
Factores de riesgo, Diagnóstico y Tratamiento de la enfermedad de LeggCalvé-Perthes

Vol. 3, núm. 3 Esp., (2019)

Alex William Marin Cherrez; Marjorie Jacqueline Ramírez Ortiz; María Belén Zhindón Astudillo; Javier Fabricio Valdez Zambrano

$$
\text { (c) (i) (9) }
$$

\section{RECONOCIMIENTO-NOCOMERCIAL-COMPARTIRIGUAL}

CC BY-NC-SA

ESTA LICENCIA PERMITE A OTROS ENTREMEZCLAR, AJUSTAR Y CONSTRUIR A PARTIR DE SU OBRA CON FINES NO COMERCIALES, SIEMPRE Y CUANDO LE RECONOZCAN LA AUTORÍA Y SUS NUEVAS CREACIONES ESTÉN BAJO UNA LICENCIA CON LOS MISMOS TÉRMINOS. 